\title{
Topografia da artéria hepática no fígado de capivara (Hydrochaerus hydrochaeris)
}

\author{
Arrangement of hepatic artery in capybara (Hydrochaerus hydrochaeris)
}

\author{
Wilson Machado de Souza' ${ }^{I}$ Nair Trevizan Machado de Souza \\ Roberto Gameiro de Carvalho ${ }^{\mathrm{I}}$ \\ Cláudia Neves Correa ${ }^{I}$
}

\section{RESUMO}

A distribuição dos ramos da artéria hepática, no fígado, foi estudada em 30 fígados de capivara (Hydrochaerus hydrochaeris) mediante injeção arterial com látex natural corado, fixação em formol a $10 \%$, dissecção pela face visceral $e$ esquematização. A lobação do fígado, nestes animais, é semelhante à do suíno, permitindo identificar os lobos lateral direito, medial direito, quadrado, medial esquerdo, lateral esquerdo e caudado (processos caudado e papilar). A artéria hepática divide-se mais freqüentemente $(73,3 \%)$ nos ramos direito e esquerdo e, em menor número de preparações (26,6\%), trifurca-se nos ramos direito, intermédio e esquerdo. Esses vasos alcançam, sob diferentes arranjos, os lobos do fígado.

Palavras-chave: fígado; capivara; artéria hepática; roedor; anatomia.

\section{ABSTRACT}

The distribution of the hepatic arterial branches in capybara's (Hydrochaerus hydrochaeris) liver was studied in 30 animals. After arterial injection with stain natural latex, the organs were fixed in formaldehyde $10 \%$, dissected and schematized. The lobation of the liver in these animals, is similar to the one of the swine, allowing to identify the following lobes: right lateral, right medial, quadrate, left medial, left lateral and caudate (processes caudate and papillary). The hepatic artery is divided more frequently (73.3\%) in the branches right and left and in lesser number of preparations (26.6\%), the vessel gives three resulting in the branches right, intermediate and left. These vessels reach, under different arrangements the lobes of the liver, when appears coincident passages with the one of the biliferous ways and portal vein, allowing in this way establish an port-bilio-arterial division.

Key words: liver; capybara; hepatic artery; rodent; anatomy.

\section{INTRODUÇÃO}

Os roedores são mamíferos que se caracterizam pela alta capacidade reprodutiva e pela baixa especificidade alimentar. Particularmente na capivara, esses fatores, em nosso meio, têm permitido, por vezes, a multiplicação indiscriminada da espécie, gerando uma competição com os animais domésticos. Por outro lado, esses vertebrados vêm apresentando uma crescente domesticação e já são explorados como produtores de carne e, em algumas propriedades, essa criação apresenta práticas de manejo com etapas bem definidas.

Esses mamíferos demonstram também, além do potencial zootécnico, a possibilidade de aproveitamento como modelos experimentais, especialmente nas áreas de produção, reprodução e cirurgia. Nesse sentido, o arranjo dos elementos hepáticos no fígado permite a divisão do órgão em unidades designadas segmentos anátomo-cirúrgicos (COINAUD, 1981; DI DIO, 2002).

De fato, a disposição dos componentes hepáticos, ou seja, vasos , nervos, ductos e tecido conjuntivo, sugere a divisão do órgão em unidades menores que, pela sua relevância, já foi analisada em várias oportunidades. JULIAN \& DeOME, (1949) estudaram-na em bovinos; JULIAN, (1952), em fetos de bovinos e de cavalos; ARNAUTOVIC \& KREMAR, (1964), SANTOS et al., (1990), em ovinos; FEITOSA FILHO, (1972), e BRIKAS \& TSIAMITAS, (1980), na

\footnotetext{
IDepartamento de Apoio, Produção e Saúde Animal, Faculdade de Odontologia de Araçatuba, Universidade Estadual Paulista (UNESP). Rua Clóvis Pestana nº 793, CP 341, 16050-680, Araçatuba, SP, Brasil. E-mail: souzawm@fmva.unesp.br.*Autor para correspondência.
} 
cabra, como também, a relação do fígado com a veia cava caudal; NASCIMENTO e GODINHO, (1960-61), no ovino e PASSAFARO et al. (1997), no bovino.

Particularizando a artéria hepática, encontram-se os trabalhos de NETTELBLAD, (1954) em crícetos; VILLIN, (1965), em roedores, BOULOGNE, (1972), em suíno; PRADA et al. (1976), em caprino e KNASIECKA, (1988), na raposa. Relativamente à capivara, destacam-se os informes de MIGLINO et al. $(1986,1997)$ e os de SOUZA et al. (1999). Este trabalho objetiva estabelecer, neste roedor, a disposição dos vasos arteriais em relação aos diferentes lobos do fígado na capivara, procurando evidenciar uma divisão portobilio-arterial.

\section{MATERIAL E MÉTODOS}

No presente estudo, foram utilizados 30 fígados de capivara (Hydrochaerus hydrochaeris), machos e fêmeas, adultos, oriundos dos municípios de Salmourão e Guararapes, e abatidos em Iguape, Estado de São Paulo. Esses órgãos foram obtidos durante evisceração dos animais, quando se tomou o cuidado de preservar íntegros os vasos relativos às vísceras abdominais.

A artéria hepática, em cada uma das peças, foi dissecada em seu trato inicial e, em correspondência à sua distribuição no fígado, foi injetada com Neoprene látex 450. Em seguida, as peças foram fixadas em solução de formol a $10 \%$, dissecadas pela face visceral, fotografadas e esquematizadas. Na divisão do fígado da capivara, adotamos os critérios estabelecidos por NICKEL et al. (1981) relativos aos animais domésticos em geral, obedecendo às indicações da ICVGAN (1994).

\section{RESULTADOS}

A morfologia externa do fígado da capivara (Hydrochaerus hydrochaeris) é semelhante à do suíno, permitindo identificar os lobos lateral direito, medial direito, quadrado, medial esquerdo, lateral esquerdo e caudado, com os processos papilar e caudado, aos quais destinam-se, sob diferentes arranjos, os ramos da artéria hepática (Figura 1).

A vesícula biliar posiciona-se entre os lobos quadrado e medial direito, quase totalmente fusionados

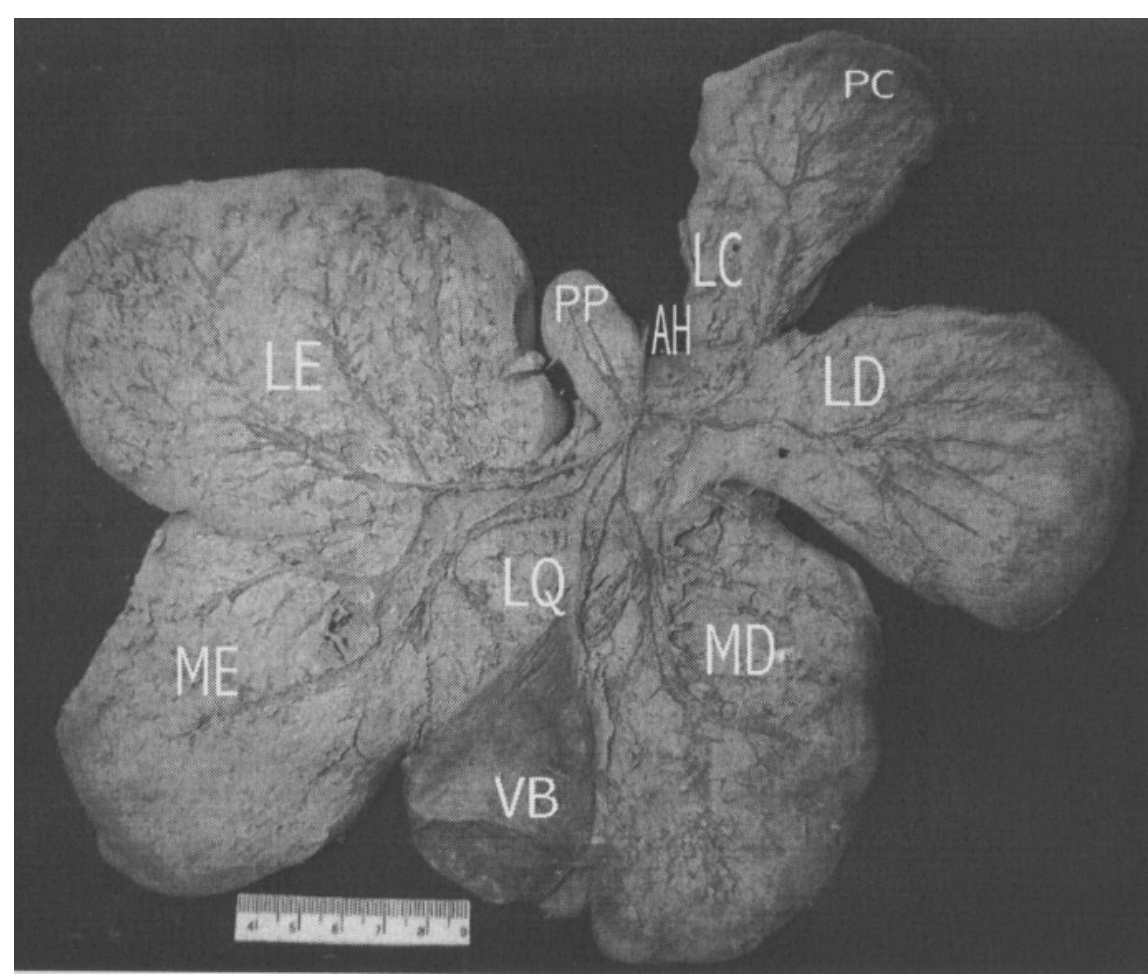

Figura 1 - Fotografia da face visceral do fígado de capivara (Hydrochaerus hydrochaeris), cujos vasos arteriais foram injetados, a partir da artéria hepática (AH), com Neoprene Látex. Observa-se a sua distribuição nos lobos lateral esquerdo (LE), medial esquerdo (ME), quadrado (LQ), medial direito (MD), lateral direito (LD) e lobo caudado (LC), com seus respectivos processos papilar (PP) e caudado (PC), além da vesícula biliar (VB).

Ciência Rural, v.37, n.1, jan-fev, 2007. 
na espécie, sem determinar uma marcada impressão (Figura 1).

A artéria hepática, no fígado da capivara, bifurca-se (Figura 2) nos ramos direito e esquerdo 22 vezes $(73,3 \% \pm 8,1)$ ou trifurca-se (Figura 3 ) nos ramos, direito, intermédio e esquerdo 8 vezes $(26,6 \% \pm 8,1)$.

O ramo esquerdo da artéria hepática está representado, apenas pelo vaso correspondente ao lobo lateral esquerdo 5 vezes $(16,6 \% \pm 6,8)$; pelos ramos destinados aos lobos lateral esquerdo, medial esquerdo, quadrado e medial direito, 10 vezes $(33,3 \% \pm 8,6)$; medial esquerdo e quadrado, 4 vezes $(13,3 \% \pm 6,2)$; lateral esquerdo, medial esquerdo, quadrado e medial direito, 4 vezes $(13,3 \% \pm 6,2)$; lateral esquerdo, medial esquerdo, lateral direito e caudado (processos papilar e caudado), duas vezes $(6,6 \% \pm 4,5)$; lateral esquerdo, medial direito e caudado (processo papilar e caudado), duas vezes $(6,6 \% \pm 4,5)$; lateral esquerdo, medial esquerdo, quadrado, medial direito e lateral direito, duas vezes $(6,6 \% \pm 4,5)$; lateral esquerdo, medial esquerdo e quadrado, uma vez $(3,3 \% \pm 3,3)$.

O ramo intermédio da artéria hepática identificado naquelas peças onde este vaso trifurca-se (Figura 2) apresenta o seguinte comportamento: divide-

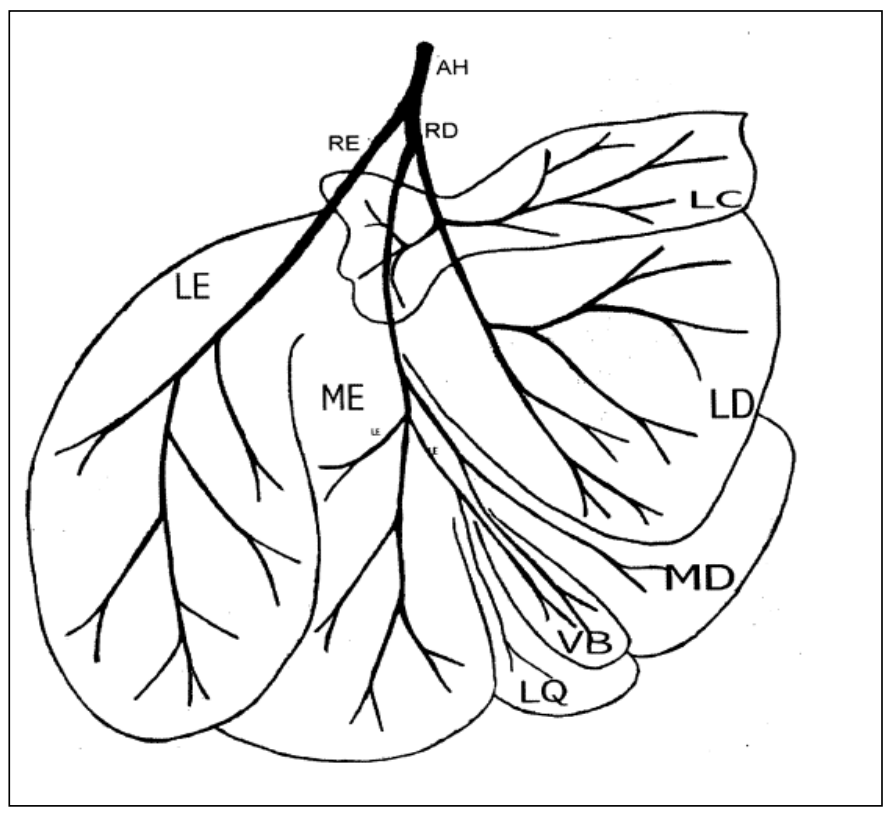

Figura 2 - Esquema representativo da face visceral do fígado de capivara (Hydrochaerus hydrochaeris), onde identificamos os lobos lateral esquerdo (LE), medial esquerdo (ME), quadrado (LQ), medial direito (MD), lateral direito (LD) e lobo caudado (LC), além da vesícula biliar (VB). A artéria hepática $(\mathrm{AH})$ divide-se nos ramos direito (RD) e esquerdo (RE); o primeiro alcança os lobos medial esquerdo, quadrado, medial direito, lateral direito e caudado e o segundo ramo atinge apenas o lobo lateral esquerdo. se nos ramos endereçados aos lobos medial esquerdo, quadrado e medial direito, 4 vezes $(13,3 \% \pm 6,2)$ ou está representado apenas pela artéria cística, responsável pela vascularização da vesícula biliar e ducto cístico, 4 vezes $(13,3 \% \pm 6,2)$.

O ramo direito da artéria hepática resulta da associação dos vasos destinados aos lobos medial esquerdo, quadrado, medial direito, lateral direito e caudado (processos papilar e caudado), 8 vezes (26,6\% $\pm 8,1$ ); lateral direito e caudado (processos papilar e caudado), 10 vezes $(33,3 \% \pm 8,6)$; lateral esquerdo, medial direito, lateral direito e caudado (processos papilar e caudado), 4 vezes (13,3\% $\pm 6,2)$; medial direito, lateral direito e caudado (processos papilar e caudado), 3 vezes (10,0\% $\pm 5,5)$; quadrado e medial direito, duas vezes $(6,6 \% \pm 4,5)$; medial esquerdo, quadrado e ramo cístico, duas vezes $(6,6 \% \pm 4,5)$; caudado (processos papilar e caudado), uma vez $(3,3 \% \pm 3,3)$.

\section{DISCUSSÃO}

As estruturas componentes dos pedículos hepáticos, ou seja, as vias bilíferas, a artéria hepática e a veia porta, por um lado, e as veias hepáticas de outro, além de seus papéis funcionais nas múltiplas atividades do fígado, são componentes estabilizadores do órgão conforme é possível verificar mediante os resultados demonstrados por JULIAN e DeOME, (1949), em bovinos; JULIAN, (1952), em fetos de bovinos e cavalos; ARNAUTOVIC e KREMAR, (1964), e SANTOS et al. (1990), na cabra, NASCIMENTO e GODINHO, (1960-61), e PASSAFARO et al. (1997), em bovinos; SOUZA et al. (1999) na capivara, e como informam COINAUD, (1981) e DI DIO(2002), orientam uma segmentação desta glândula, objetivando uma possível hepatectomia.

Esses autores, ao exporem seus resultados, aludem direta ou indiretamente à possibilidade de divisão territorial do fígado conforme a distribuição vascular, estabelecendo limites paucivasculares no parênquima do órgão.

Ao cuidar da distribuição intraparenquimatosa da artéria hepática, NETTELBLAD, (1954) em crícetos, estabelece uma divisão lobar do fígado deste mamífero, que irá fundamentar trabalhos subseqüentes em outros animais. Deste modo, VILLIN (1965), em ratos e cobaias, registra que as artérias hepáticas são variáveis e se distribuem no fígado conforme 


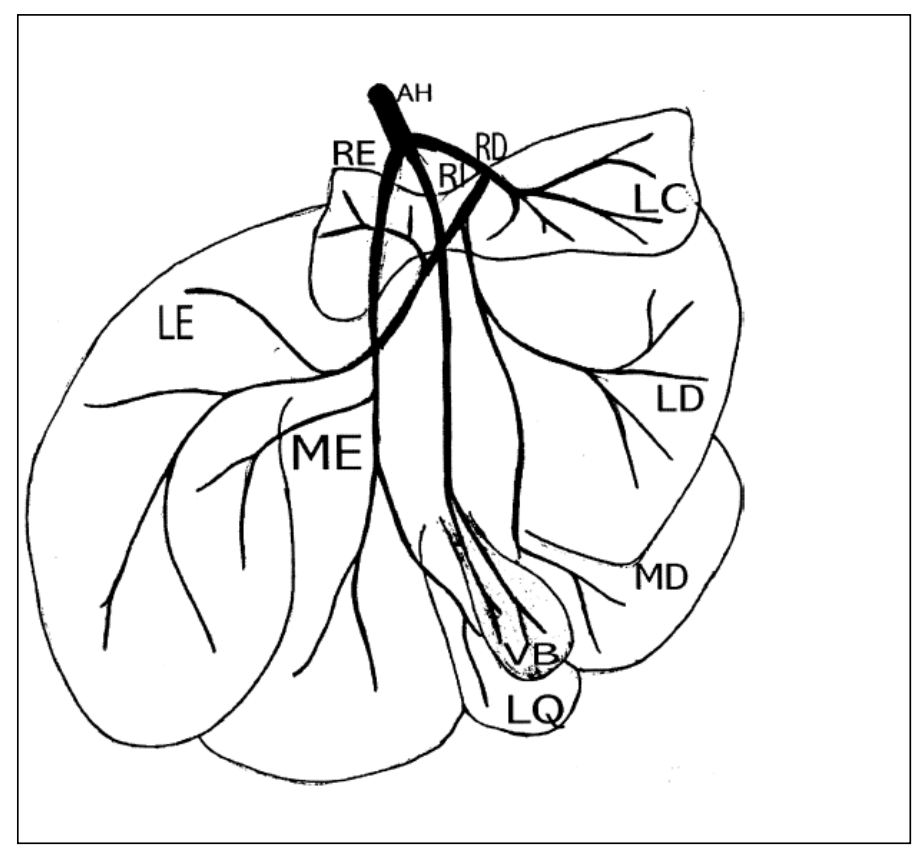

Figura 3 - Esquema da face visceral do fígado de capivara (Hydrochaerus hydrochaeris), onde identificamos os lobos lateral esquerdo (LE), medial esquerdo (ME), quadrado (LQ), medial direito (MD), lateral direito (LD) e lobo caudado (LC), além da vesícula biliar (VB). A artéria hepática (AH) divide-se nos ramos direito (RD), intermédio (RI) e esquerdo (RE). O ramo direito alcança os lobos caudado (processos caudado e papilar), lateral direito, medial direito e lateral esquerdo. O RI distribui-se apenas na vesícula biliar e ducto colédoco e o RE atinge os lobos lateral esquerdo, medial esquerdo e quadrado.

três sistemas principais (direito, intermédio e esquerdo), alusões que provavelmente coincidem com algumas observações $(26,6 \%)$ da capivara, nas quais registramos o referido vaso trifurcando-se nos ramos direito, intermédio e esquerdo; portanto, o comportamento menos freqüente neste vertebrado.

Verificando o assunto no suíno, animal que apresenta o fígado morfologicamente semelhante ao da capivara, BOULOGNE (1972) assinala que a distribuição da artéria hepática no fígado obedece aos trajetos dos vasos portais e das vias bilíferas, acompanhando a segmentação estabelecida por esses vasos, resultados já anteriormente apresentados na capivara por MIGLINO et al. (1986 e 1997), ao estudarem a disposição dessas estruturas, e que não se afastam das observações deste trabalho em relação à artéria hepática. BOULOGNE (1972), ao expor seus resultados, apresenta esquemas de distribuição segmentar desse vaso semelhantes ao que se verifica na capivara, onde registra-se a artéria hepática mais freqüentemente (73,3\%) oferecendo dois ramos, esquerdo e direito, ou dividindo-se simultaneamente (26,6\%) em três vasos (direito, intermédio e esquerdo). Em ambas as modalidades e sob diferentes arranjos, os vasos destinam-se aos lobos lateral direito, medial direito, quadrado, medial esquerdo, lateral esquerdo e caudado (processos papilar e caudado) do fígado.

Relativamente ao comportamento da artéria hepática no fígado de caprinos, PRADA et al. (1976) registram a distribuição desse vaso conforme dois ramos que alcançam os setores medial, dorsal, intermédio e lateral do lobo direito, além dos processos caudado do lobo caudado e à vesícula biliar e outro aos territórios medial, intermédio e lateral do lobo esquerdo, além do lobo quadrado. por outro lado, KNASIECKA (1988), na raposa vermelha, refere uma distribuição vascular da artéria hepática a partir de um (60,0\%), dois (33,3\%) e três $(6,7 \%)$ ramos. Possivelmente a diferença de comportamento desses vasos decorra da lobação hepática, pois os ruminantes mostram fígado contínuo, enquanto, nos carnívoros, esse órgão é lobado. Na capivara, a lobação é semelhante à observada no suíno, ou seja, a divisão lobar está presente, porém de uma maneira mais discreta que nos carnívoros e a distribuição vascular arterial se processa mediante dois $(73,3 \%)$ ou três ramos (26,6\%), conforme alusão dos diversos autores a respeito do comportamento das vias bilíferas e da veia porta.

\section{CONCLUSÕES}

Por meio do exame da distribuição da artéria hepática nos órgãos estudados, verifica-se que sua morfologia é semelhante à do fígado do suíno, sendo possível identificar os lobos lateral direito, medial direito, quadrado, medial esquerdo, lateral esquerdo e caudado (processos caudado e papilar), aos quais a artéria hepática envia, sob diferentes arranjos, os ramos resultantes de sua divisão. Nessas preparações, a vesícula biliar posiciona-se entre os lobos quadrado e medial direito, sem determinar uma marcada impressão.

A artéria hepática bifurca-se nos ramos direito e esquerdo com maior freqüência (73,3\%) ou trifurca-se nos ramos direito, intermédio e esquerdo (26,6\%). O ramo esquerdo está representado apenas pelo vaso correspondente ao lobo lateral esquerdo (16,6\%); aos lobos lateral esquerdo, medial esquerdo, quadrado e medial direito (33,3\%); medial esquerdo e quadrado (13,3\%); lateral esquerdo, medial esquerdo, 
lateral direito e caudado (processos papilar e caudado) $(6,6 \%)$; lateral esquerdo, medial direito e caudado (processo papilar e caudado) (6,6\%); lateral esquerdo, medial esquerdo, quadrado, medial direito e lateral direito (6,6\%); lateral esquerdo, medial esquerdo e quadrado (3,3\%).

O ramo intermédio da artéria hepática, onde esse vaso trifurca-se, divide-se nos ramos endereçados aos lobos medial esquerdo, quadrado e medial direito (13,3\%) ou apenas pela artéria cística (13,3\%). O ramo direito da artéria hepática resulta da associação dos vasos destinados aos lobos medial esquerdo, quadrado, medial direito, lateral direito e caudado (26,6\%); lateral direito e caudado (33,3\%); lateral esquerdo, medial direito, lateral direito e caudado (13,3\%); medial direito, lateral direito e caudado (10,0\%); quadrado e medial direito (6,6\%); medial esquerdo, quadrado e ramo cístico $(6,6 \%)$ e caudado (3,3\%).

O comportamento da artéria hepática nesses roedores acompanha a disposição da veia porta e das vias bilíferas, constituindo a divisão porto-bilio-arterial, referida com freqüência pelos diversos autores que cuidaram do tema.

\section{REFERÊNCIAS}

ARNAUTOVIC, I.; KREMAR, I. A contribution to the knowledge of the intrahepatic blood vessels and gall-ducts in sheep. Veterinaria Sarajievo, v.13, n.1, p.15-28, 1964.

BOULOGNE, J.C. La segmentation hepatique chez lê porc domestique (Sus scrofa domesticus), 1972. 127f. Thèse (Doutorado em Medicina Veterinária) - École National Vétérinaire D’Alfort.

BRIKAS, P.; TSIAMITAS, C. Anatomic arrangement of the hepatic veins in the goat. Am J Vet Res, v.41, n.5, p.796797, 1980.

COINAUD, C. Controlled hepatectomies and exposure af the intrahepatic bile ducts - Anatomical and technical study. Paris: Masson, 1981. 112p.

DI DIO, L.J.A. Tratado de anatomia aplicada. São Paulo: Atheneu, 2002. 948p.

FEITOSA - FILHO, E. Estudo anatômico das veias hepáticas em fígados de Capra hircus. In: CONGRESSO BRASILEIRO DE MEDICINA VETERINÁRIA, 13., 1972, Brasília, DF. Anais... Brasília, 1972. p.366.

INTERNATIONAL COMMITEE ON VETERINARY GROSS ANATOMICAL NOMENCLATURE. Nomina anatomica veterinaria. 4.ed. Zurich, 1994. (Together with nomina histologica, 2.ed. 1992 and nomina embriológica veterinária, 1992).

JULIAN, L.M. Studies on the subgroups anatomy of the bovine liver III - Comparative arrangement of the blood vessels of liver's of the bovine and equine fetuses. Am J Vet Res, v.13, n.96, p.201-203, 1952.

JULIAN, L.M.; DeOME, K.B. Studies on the subgroups anatomy of the bovine liver, I - The distribuition of the blood vessels and bile ducts as revealed by the vinylite - corrosion technique. Am J Vet Res, v.10, n.37, p.331-335, 1949.

KNASIECKA, V. Branches of the hepatic artery in the red Fox. Anat Anz Jena, v.166, p.91-96, 1988.

MIGLINO, M.A. et al. Contribuição ao estudo do sistema excretor do fígado em animais silvestres. V. Vias bilíferas da capivara (Hidrochoerus hidrochoeris). Revista da Faculdade de Medicina Veterinária e Zootecnia da Universidade de São Paulo, v.23, n.2, p.115-132,1986.

MIGLINO, M.A. et al. Distribuição da veia porta no fígado da capivara (Hidrochoerus hidrochoeris). Braz J Vet Res Anim, v.34, n.1, p.9-11, 1997.

NASCIMENTO, J.F.; GODINHO, H.P. Nota anatômica sobre as relações da veia cava caudalis e o fígado de Ovis aries. Arq Escola Vet Belo Horizonte, v.13, p.249-254, 1960/61.

NETTELBLAD, S.C. Die lobierung und innere Topographie der Säugerleber: nebst Beiträgen zur Kenntnis der Leberentwichlung kein Goldhamster (Cricetus auratus). Acta Anat, v.21, n.20, p.92-98, 1954.

NICKEL, R. et al. The anatomy of the domestic animals. The circulatory system. Berlin: Paul Parey, 1981. V.3, 610p.

PASSAFARO, R. et al. Veia cava caudal e ósteos das veias hepáticas em bovinos da raça Nelore. Braz J Vet Res Anim Sci, v.34, n.2, p.70-72, 1997

PRADA, I.L.S. et al. Contribuição ao estudo do comportamento da artéria hepática em caprinos. In: CONGRESSO BRASILEIRO DE ANATOMIA, 9., CONGRESSO LUSO-BRASILEIRO DE ANATOMIA, 2., 1976, Niterói, RJ. Anais... Niterói, 1976. p.199.

SANTOS, R.M.B. et al. Mayor and minor hepatic veins liver segmentation in ovine (Ovis aries). Braz J Vet Res Anim Sci, v.28, n.1, p.19-30, 1990.

SOUZA, W.M. et al. The hepatic veins of capybara (Hidrochoerus hidrochoeris). Braz J morphol Sci, v.16, n.2, p.181-184, 1999.

VILLIN, B. Topographie vascularie et biliaire du foie de quelques Rongeurs domestiques. 1965. 80f. Thèse (Doutorado em Medicina Veterinária) - École Nationale Vétérinaire D’Alfort. 